\title{
Steric Effects Govern the Photoactivation of Phytochromes
}

\author{
Olle Falklöf and Bo Durbeej
}

\section{Linköping University Post Print}

\section{Tweet}

N.B.: When citing this work, cite the original article.

Original Publication:

Olle Falklöf and Bo Durbeej, Steric Effects Govern the Photoactivation of Phytochromes, 2016, ChemPhysChem, (17), 7, 954-957.

http://dx.doi.org/10.1002/cphc.201501080

Copyright: Wiley-VCH Verlag

http://www.wiley-vch.de/publish/en/

Postprint available at: Linköping University Electronic Press

http://urn.kb.se/resolve?urn=urn:nbn:se:liu:diva-127554 


\section{Steric Effects Govern the Photoactivation of Phytochromes}

\section{Olle Falklöf and Bo Durbeej ${ }^{*[a]}$}

Phytochromes constitute a superfamily of photoreceptor proteins that exist in two forms that absorb red (Pr) and far-red (Pfr) light. Although it is well-known that the conversion of Pr into Pfr (the biologically active form) is triggered by a $Z \rightarrow E$ photoisomerization of the linear tetrapyrrole chromophore, direct evidence is scarce as to why this reaction always occurs at the methine bridge between pyrrole rings $\mathrm{C}$ and $\mathrm{D}$. Here, we present hybrid quantum mechanics/molecular mechanics calculations based on a highresolution $\mathrm{Pr}$ crystal structure of Deinococcus radiodurans bacteriophytochrome to investigate the competition between all possible photoisomerizations at the three different (AB, BC and $C D$ ) methine bridges. The results demonstrate that steric interactions with the protein are a key discriminator between the different reaction channels. In particular, it is found that such interactions render photoisomerizations at the $A B$ and $B C$ bridges much less probable than photoisomerization at the $C D$ bridge.

Phytochromes constitute a widespread superfamily of photoreceptor proteins responsive to red and far-red light that were first discovered in plants but are present also in other organisms such as bacteria, cyanobacteria and algae. ${ }^{[1-3]}$ In response to the prevailing light conditions, these photoreceptors regulate physiological and developmental processes in their host organisms by interconverting between two photochromic forms: a red-light absorbing Pr form and a far-red-light absorbing Pfr form. ${ }^{[4]}$ In most phytochromes, the equilibrium between $\mathrm{Pr}$ and $\mathrm{Pfr}$ is shifted toward $\mathrm{Pr}$ in the dark-adapted state and toward $\mathrm{Pfr}$ in the biologically active state. ${ }^{[5-8]}$ The red-light-induced conversion of $\mathrm{Pr}$ into $\mathrm{Pfr}$ is triggered by a $Z \rightarrow E$ photoisomerization of the linear tetrapyrrole (bilin) chromophore (Figure 1) and proceeds via a number of metastable protein intermediates. ${ }^{[5-8]}$ Today, although direct evidence is scarce, it is widely accepted that the photoisomerization occurs at the C15$C 16$ bond of the methine bridge between pyrrole rings $C$ and $D$, thus corresponding to a rotation of ring $D$ around this bond. ${ }^{[9-22]}$ Such a mechanism is supported by X-ray crystallographic data ${ }^{[9-}$ $13,15,16,19,21]$ and studies using a variety of spectroscopic techniques such as circular dichroism, ${ }^{[14]}$ resonance Raman $(\mathrm{RR}),{ }^{[17]}$ femtosecond visible pump infrared probe, ${ }^{[18]}$ magic angle spinning $\mathrm{NMR}^{[20]}$ and femtosecond to microsecond transient absorption ${ }^{[22]}$ spectroscopy. For example, crystal structures of $\mathrm{Pr}$ forms of bacterial, ${ }^{[9-11,16,21]}$ cyanobacterial ${ }^{[12,15]}$ and plant ${ }^{[19]}$ phytochromes show that while rings $A-C$ are tightly packed by the surrounding protein, ring $D$ resides in a cavity that seems to more easily accommodate a rotation around the C15C16 bond. Furthermore, in contrast to solution-phase NMR data on Synechococcus OS-B' (a cyanobacterium) phytochrome that, a few years ago, were taken as support for an alternative mechanism in which the photoisomerization rather involves a rotation of ring $A,{ }^{[23]}$ recently reported magic angle spinning NMR data on the same phytochrome are indeed consistent with the

[a] O. Falklöf, Prof. B. Durbee

Division of Theoretical Chemistry, IFM, Linköping University SE-581 83 Linköping, Sweden

E-mail: bodur@ifm.liu.se

Supporting information for this article is available on the WWW under X. view that the photoisomerization takes place at the $\mathrm{C} 15-\mathrm{C} 16$ bond. [20]

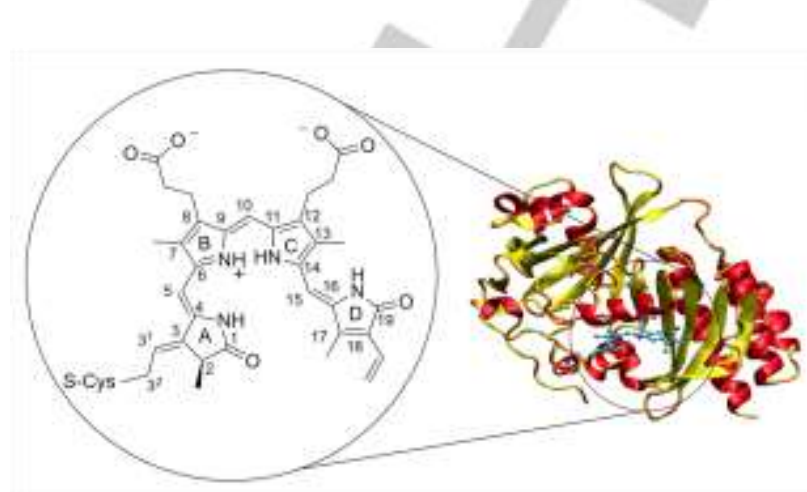

Figure 1. Structure of the biliverdin IXa chromophore and the chromophore binding domain of Deinococcus radiodurans bacteriophytochrome (DrBphP) in the Pr form. The chromophore is covalently linked to Cys24 of the apoprotein.

In the absence of a protein environment, on the other hand, it has been shown using both quantum chemical calculations at different levels of theory ${ }^{[24-26]}$ and nonadiabatic surface hopping dynamics simulations ${ }^{[27]}$ that bilin chromophores are intrinsically more prone to undergo a $Z \rightarrow E$ photoisomerization at the $C 10$ $\mathrm{C} 11$ bond between rings $\mathrm{B}$ and $\mathrm{C}$, than at the $\mathrm{C} 15-\mathrm{C} 16$ bond between rings $C$ and $D$. Indirectly, this finding suggests that phytochromes rely on interactions between the chromophore and the protein not only to facilitate a very fast photoreaction, ${ }^{[28]}$ but also to ensure that the reaction takes place at the C15-C16 bond. While previous computational studies have investigated the impact of such interactions on UV-vis absorption ${ }^{[2,29]}$ and $\mathrm{RR}^{[30]}$ spectra of phytochromes, it has not been established how the protein exerts control over the possible photoreactions of the chromophore. Here, we report hybrid quantum mechanics/molecular mechanics $(\mathrm{QM} / \mathrm{MM})^{[31]}$ calculations based on a high-resolution (1.45 $\AA$ ) $\mathrm{Pr}$ crystal structure of the chromophore binding domain of Deinococcus radiodurans bacteriophytochrome ${ }^{[10]}$ (DrBphP, Figure 1) to show that steric effects play a decisive role for the $\mathrm{Pr} \rightarrow \mathrm{Pfr}$ activation of phytochromes. In fact, as we will see, steric effects are found to be a key discriminator between possible photoisomerization pathways.

As further outlined in the Computational Methods section of the Supporting Information (see also associated Table S1 and Figure S1), all calculations were carried out with a $\mathrm{QM} / \mathrm{MM}$ scheme combining time-dependent density functional theory (TD-DFT) ${ }^{[32]}$ with the AMBER force field ${ }^{[33]}$ that we recently found is able to reproduce the experimental UV-vis absorption maxima (Soret and $\mathrm{Q}$ bands) of $\operatorname{Pr}$ DrBphP with an accuracy of about $0.15 \mathrm{eV}^{[29]}$ In brief, the $\omega B 97 X-D^{,[34]}$ CAM-B3LYP ${ }^{[35]}$ and LC$\mathrm{BLYP}^{[36]}$ range-separated hybrid density functionals were used to model the biliverdin IXa (BV) chromophore and Cys24 side chain included in the $\mathrm{QM}$ region (Figure 1), and $\mathrm{AMBER}^{[33]}$ provided the MM description of the surrounding protein, with socalled electronic embedding between the QM and MM layers. ${ }^{[37]}$ 


\begin{tabular}{|c|c|c|c|c|c|c|c|}
\hline & & AB bridg & & $\mathrm{BC}$ bridge & & CD bridge & \\
\hline QM functional & state & C4-C5 & $\mathrm{C} 5-\mathrm{C} 6$ & C9-C10 & C10-C11 & C14-C15 & C15-C16 \\
\hline \multirow[t]{2}{*}{$\omega B 97 X-D$} & $\mathrm{~S}_{0}$ & 1.36 & 1.41 & 1.37 & 1.41 & 1.45 & 1.35 \\
\hline & $\mathrm{S}_{1}$ & 1.38 & 1.40 & 1.39 & 1.40 & 1.41 & 1.37 \\
\hline \multirow[t]{2}{*}{ CAM-B3LYP } & $\mathrm{S}_{0}$ & 1.36 & 1.41 & 1.37 & 1.41 & 1.44 & 1.34 \\
\hline & $\mathrm{S}_{1}$ & 1.38 & 1.40 & 1.39 & 1.40 & 1.41 & 1.37 \\
\hline \multirow[t]{2}{*}{ LC-BLYP } & $\mathrm{S}_{0}$ & 1.35 & 1.41 & 1.35 & 1.41 & 1.45 & 1.33 \\
\hline & $\mathrm{S}_{1}$ & 1.37 & 1.40 & 1.39 & 1.39 & 1.42 & 1.35 \\
\hline
\end{tabular}

As an initial assessment of how the chromophore responds to light absorption, we first investigated its geometric relaxation from the vertical Franck-Condon (FC) point on the lowest excited singlet state $\left(S_{1}\right)$ potential energy surface (PES) populated by red light. This was done by performing geometry optimizations in both the ground state $\left(\mathrm{S}_{0}\right)$ and the excited state. Focusing on the geometric changes of the three methine bridges, these results are summarized in Table 1.

First, we note that all methine bridges consistently show a distinct differentiation between single (C5-C6, C10-C11 and C14-C15) and double (C4-C5, C9-C10 and C15-C16) bonds in the ground state. Specifically, the differences in lengths between the two bonds of each methine bridge are at least $0.05,0.04$ and $0.10 \AA$ for the $A B, B C$ and $C D$ bridges, respectively. Furthermore the bond of each bridge that has more single-bond character than the other in the ground state (i.e., C5-C6, C10-C11 and $\mathrm{C} 14-\mathrm{C} 15)$ is shortened during the FC relaxation, which means that light absorption does not at all facilitate isomerization around these bonds. In other words, it seems unlikely that the photoreaction of $\operatorname{Pr}$ DrBphP occurs at any of these sites; rather, this process would have to involve either of the C4-C5, C9-C10 and $\mathrm{C} 15-\mathrm{C} 16$ bonds, each of which is indeed lengthened (by 0.02-0.04 $\AA$ ) during the FC relaxation. Already from this analysis it can be inferred that interactions with the protein have a distinct influence on the photochemical reactivity of the chromophore, because without such interactions, the same type of $Q M$ methods that herein are used for the $\mathrm{QM} / \mathrm{MM}$ calculations have previously predicted that the FC relaxation of $\mathrm{BV}$ is substantially dominated by stretching of $(>0.04 \AA)$ and torsional motion around $\left(>10^{\circ}\right)$ the $\mathrm{C} 10-\mathrm{C} 11$ bond. ${ }^{[26]}$

To assess which of the C4-C5, C9-C10 and C15-C16 bonds is the preferred site for $Z \rightarrow E$ photoisomerization, we then mapped the regions of the excited-state PES for torsional motion around these bonds, as described by the C3-C4-C5-C6, C8-C9C10-C11 and C14-C15-C16-C17 dihedral angles, respectively. Specifically, starting from the FC point and covering up to $\pm 50^{\circ}$ rotation around each bond relative to the $F C$ point, a series of constrained excited-state geometry optimizations were performed in which, in steps of $10^{\circ}$, a single dihedral constraint was enforced for the BV chromophore and all other degrees of freedom of the chromophore binding pocket ${ }^{[10,29]}$ were relaxed. To possibly strengthen the conclusion from Table 1 that the $\mathrm{C} 10$ $\mathrm{C} 11$ bond is less reactive than the C4-C5, C9-C10 and C15-C16 bonds, torsional motion around $\mathrm{C} 10-\mathrm{C} 11$ was also considered, as described by the $\mathrm{C} 9-\mathrm{C} 10-\mathrm{C} 11-\mathrm{C} 12$ dihedral angle. The preferred direction of torsional motion during each of the four photoisomerizations (toward $+50^{\circ}$ relative to the $\mathrm{FC}$ point for the reactions at $\mathrm{C} 9-\mathrm{C} 10$ and $\mathrm{C} 15-\mathrm{C} 16$; toward $-50^{\circ}$ relative to the FC point for the reactions at C4-C5 and C10-C11) is deduced from results given in Figures S2-S5 of the Supporting Information.

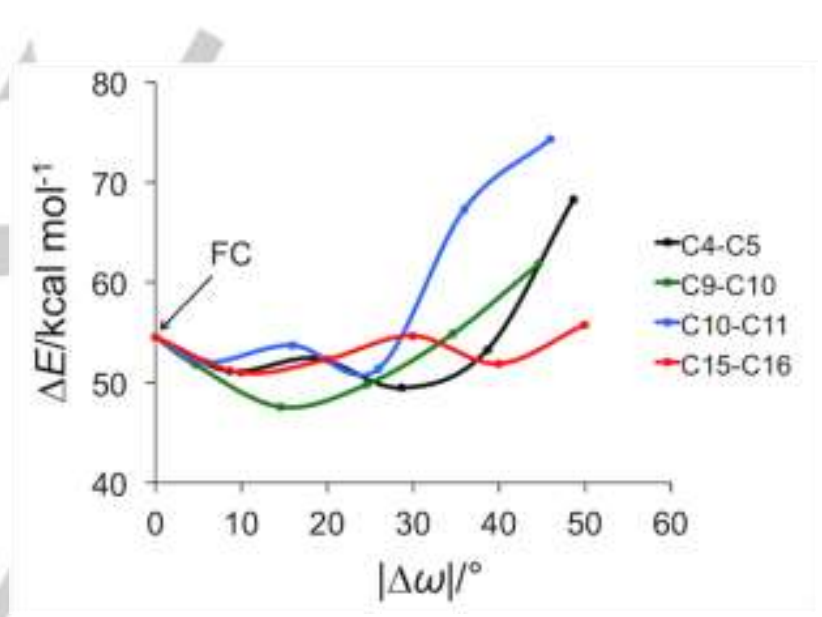

Figure 2. $\omega B 97 X-D / A M B E R$ excited-state potential energy curves for the initial stages of photoisomerization at the C4-C5, C9-C10, C10-C11 and C15-C16 bonds of $\mathrm{BV}$ in $\operatorname{Pr} D r B p h P$. Energies $(\Delta E)$ and dihedral angles $(|\Delta \omega|)$ are given relative to their ground-state $\operatorname{Pr}$ values.

The resulting potential energy curves are presented in Figure 2 ( $\omega B$ B7X-D results) and in Figures S6 (CAM-B3LYP) and S7 (LC-BLYP) of the Supporting Information. Since all three QM levels support the same overall conclusions, we will in the interest of brevity focus on the $\omega B 97 X-D$ results. Before discussing these, however, we note that there are two reasons why no attempt was made to include in the modeling also the regions of the excited-state PES where (closer to $\pm 90^{\circ}$ ) it has been suggested the system decays to the ground state through a conical intersection. ${ }^{[24,38,39]}$ First, as we will see, already the regions covering up to $\pm 50^{\circ}$ torsional motion relative to the $\mathrm{FC}$ point provide very valuable information on the in situ reactivity of the chromophore. Second, because of the onset of inevitable near-degeneracy effects, it is a major challenge to describe the regions where the system decays to the ground state using conventional TD-DFT. ${ }^{[40]}$ That such effects are minor for 
torsional motion of bilin chromophores of the current magnitudes has been established by comparing (for a reduced chromophore model comprising rings $\mathrm{B}$ and $\mathrm{C}$ only) the excited-state PES obtained with TD-DFT with that obtained from complete active space self-consistent field calculations, which properly account for near-degeneracy effects. ${ }^{[25]}$

Interestingly, the potential energy curves in Figure 2 give a clear indication that $\mathrm{C} 15-\mathrm{C} 16$ is the preferred site for $Z \rightarrow E$ photoisomerization, because the corresponding curve is considerably flatter than the $\mathrm{C} 4-\mathrm{C} 5, \mathrm{C} 9-\mathrm{C} 10$ and $\mathrm{C} 10-\mathrm{C} 11$ ones. In more quantitative terms, the energy barrier to complete the $50^{\circ}$ rotation is about $5 \mathrm{kcal} \mathrm{mol}^{-1}$ at $\mathrm{C} 15-\mathrm{C} 16$, but much larger $\left(19,14\right.$ and $23 \mathrm{kcal} \mathrm{mol}^{-1}$, respectively) at C4-C5, C9-C10 and C10-C11. The observation that $\mathrm{C} 10-\mathrm{C} 11$ has the steepest energy profile is in line with the analysis in Table 1 showing that this bond, as opposed to C4-C5, C9-C10 and C15-C16, is shortened during the FC relaxation. Studying the FC relaxation thus appears a useful approach for obtaining a quick, qualitative assessment of which bond is the most reactive one.

While Figure 2 supports the widely held and experimentally well-substantiated view that the photoreaction of phytochromes occurs at the C15-C16 bond, ${ }^{\left[{ }^{[9-22]}\right.}$ it is of course of interest to also understand why this is so, especially in light of the fact that direct experimental evidence in the matter is scarce. One possibility is that the chromophore isomerization pathways afforded by the protein for the different reactions are such that the isomerization at $\mathrm{C} 15-\mathrm{C} 16$ is more favorable in terms of the intrinsic energy of the chromophore. To test this hypothesis, QM excited-state singlepoint calculations using TD-DFT at the $\omega B 97 X-D / 6-$ $31 \mathrm{G}(\mathrm{d}, \mathrm{p})$ level of theory were performed on the $\mathrm{QM} / \mathrm{MM}$ chromophore geometries obtained in the protein along the C4C5, C9-C10 and C15-C16 photoisomerization pathways. Denoting the resulting energies of the bare chromophore as $E_{\mathrm{BV}}$, these calculations are summarized in Figure 3 (top). As can be seen, however, the intrinsic $\mathrm{C} 15-\mathrm{C} 16$ energy profile is not at all more favorable than the $\mathrm{C} 4-\mathrm{C} 5$ and $\mathrm{C} 9-\mathrm{C} 10$ ones. Hence, another mechanism must be at play.

A second possibility is that steric repulsion between the chromophore and the protein is an important factor, because at least as far as the situation in Pr prior to light absorption is concerned, available crystal structures show that rings A-C are more tightly packed by the protein than ring D. ${ }^{9-12,15,16,19,21]}$ This is illustrated in Figure S1 of the Supporting Information. To test the potential role of steric repulsion, singlepoint calculations were carried out with the AMBER force field ${ }^{[33]}$ to obtain the van der Waals energy $\left(E_{\mathrm{vdw}}\right)$ of the full chromophore-protein model for each geometry along the C4-C5, C9-C10 and C15-C16 photoisomerization pathways. The results of these calculations are presented in Figure 3 (bottom). As a complement, Figure S8 of the Supporting Information shows the $E_{\mathrm{vdw}}$ curve for up to $90^{\circ}$ rotation at $\mathrm{C} 15-\mathrm{C} 16$. Interestingly, the $E_{\mathrm{vdw}}$ cost to complete the $50^{\circ}$ rotation is $7 \mathrm{kcal} \mathrm{mol}^{-1}$ at C4-C5 and $11 \mathrm{kcal} \mathrm{mol}^{-1}$ at $\mathrm{C} 9-\mathrm{C} 10$, but is less than $1 \mathrm{kcal} \mathrm{mol}^{-1}$ at $\mathrm{C} 15-\mathrm{C} 16$. Furthermore, the $\sim 6$ and $\sim 10 \mathrm{kcal} \mathrm{mol}^{-1}$ by which the $E_{\mathrm{vdw}}$ costs thereby are larger at C4-C5 and C9-C10 help explain the $\sim 14$ and $\sim 9 \mathrm{kcal} \mathrm{mol}^{-1}$ magnitudes by which the corresponding photochemical energy barriers (Figure 2) are larger at these sites than at C15-C16. These results give a clear indication that $\mathrm{Pr}$ phytochromes rely on steric effects to control the reactivity of their bilin chromophore and prevent photoisomerizations at C4-C5 and C9-C10 from exerting a negative impact on the photoisomerization efficiency at C15-C16. In future work, we plan to investigate whether the same holds true for $\mathrm{Pfr}$ phytochromes and the possible pathways for the reverse $E \rightarrow Z$ photoisomerization. Also, it will be of interest to consider more complete (but with lower resolution) protein structures ${ }^{[21,41]}$ that include the PHY domain lacking in the high-resolution DrBphP structure $^{[10]}$ used in this work.
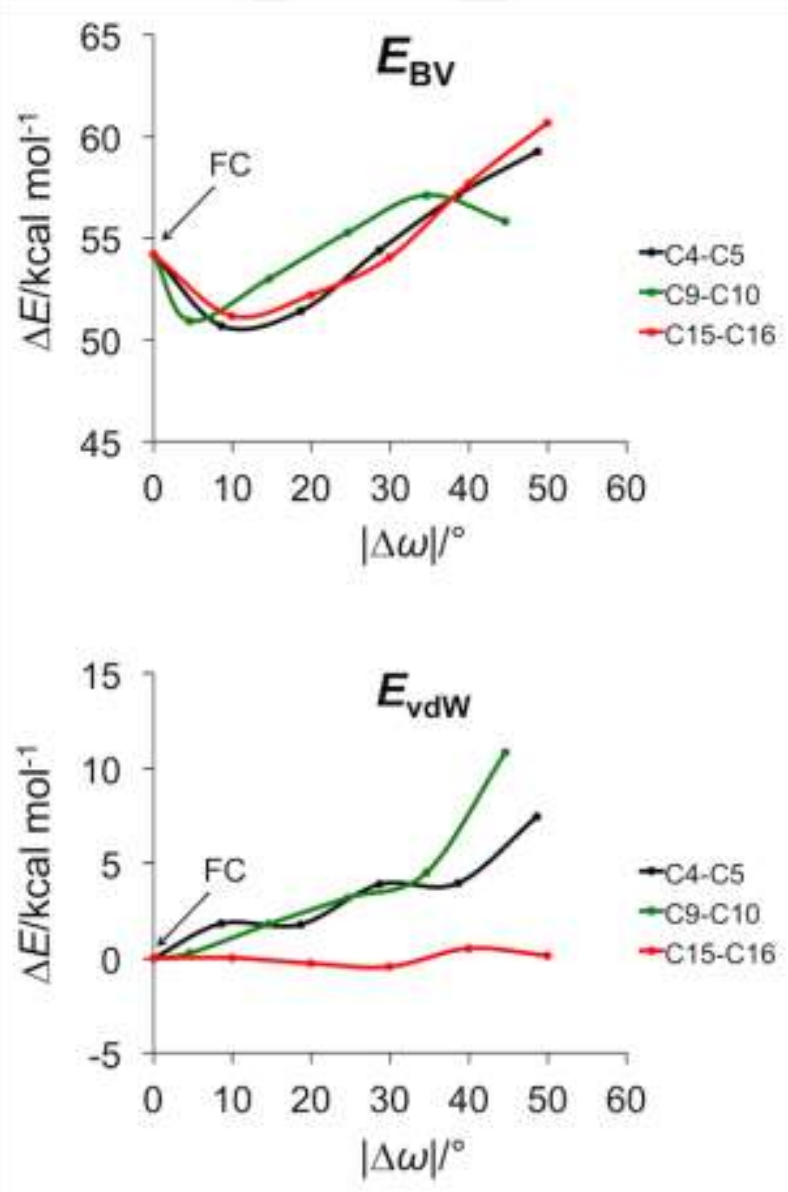

Figure 3. (Top) $\omega B 97 X-D$ excited-state potential energy curves for the bare chromophore during the initial stages of photoisomerization at the C4-C5, C9C10 and C15-C16 bonds in Pr DrBphP. (Bottom) AMBER Evaw curves for the initial stages of photoisomerization at the C4-C5, C9-C10 and C15-C16 bonds of $\mathrm{BV}$ in $\operatorname{Pr}$ DrBphP. Energies $(\Delta E)$ and dihedral angles $(|\Delta \omega|)$ are for both plots given relative to their ground-state Pr values.

\section{Acknowledgements}

We acknowledge financial support from Linköping University, the Swedish Research Council (Grant 621-2011-4353) and the Olle Engkvist Foundation, as well as grants of computing time at the Swedish National Supercomputer Centre (NSC) in Linköping. 


\section{Keywords: chromophores • isomerization • photoreceptor} proteins $\cdot \mathrm{QM} / \mathrm{MM}$ modeling $\bullet$ range-separated functionals

[1] B. Karniol, J. R. Wagner, J. M. Walker, R. D. Vierstra, Biochem. J. 2005 392, 103-116.

[2] N. C. Rockwell, D. Duanmu, S. S. Martin, C. Bachy, D. C. Price, D. Bhattacharya, A. Z. Worden, J. C. Lagarias, Proc. Natl. Acad. Sci. U. S. A. 2014, 111, 3871-3876.

[3] K. Anders, L.-O. Essen, Curr. Opin. Struct. Biol. 2015, 35, 7-16.

[4] W. Rüdiger, F. Thümmler, Angew. Chem. Int. Ed. Engl. 1991, 30 1216-1228.

[5] N. C. Rockwell, Y. S. Su, J. C. Lagarias, Annu. Rev. Plant Biol. 2006, 57, 837-858.

[6] N. C. Rockwell, J. C. Lagarias, ChemPhysChem 2010, 11, 1172-1180.

[7] A. Möglich, X. Yang, R. A. Ayers, K. Moffat, Annu. Rev. Plant. Biol. 2010, 61, 21-47.

[8] M. E. Auldridge, K. T. Forest, Crit. Rev. Biochem. Mol. Biol. 2011, 46, 67-88.

[9] J. R. Wagner, J. S. Brunzelle, K. T. Forest, R. D. Vierstra, Nature 2005 438, 325-331.

[10] J. R. Wagner, J. Zhang, J. S. Brunzelle, R. D. Vierstra, K. T. Forest, J. Biol. Chem. 2007, 282, 12298-12309.

[11] X. Yang, E. A. Stojković, J. Kuk, K. Moffat, Proc. Natl. Acad. Sci. U. S. A. 2007, 104, 12571-12576.

[12] L.-O. Essen, J. Mailliet, J. Hughes, Proc. Natl. Acad. Sci. U. S. A. 2008, 105, 14709-14714.

[13] X. Yang, J. Kuk, K. Moffat, Proc. Natl. Acad. Sci. U. S. A. 2008, 105, 14715-14720.

[14] N. C. Rockwell, L. Shang, S. S. Martin, J. C. Lagarias, Proc. Natl. Acad. Sci. U. S. A. 2009, 106, 6123-6127.

[15] J. Mailliet, G. Psakis, K. Feilke, V. Sineshchekov, L.-O. Essen, J. Hughes, J. Mol. Biol. 2011, 413, 115-127.

[16] X. Yang, Z. Ren, J. Kuk, K. Moffat, Nature 2011, 479, 428-432.

[17] K. M. Spillane, J. Dasgupta, R. A. Mathies, Biophys. J. 2012, 102, 709717.

[18] Y. Yang, M. Linke, T. von Haimberger, J. Hahn, R. Matute, L. González, P. Schmieder, K. Heyne, J. Am. Chem. Soc. 2012, 134, 1408-1411.

[19] E. S. Burgie, A. N. Bussell, J. M. Walker, K. Dubiel, R. D. Vierstra, Proc. Natl. Acad. Sci. U. S. A. 2014, 111, 10179-10184.

[20] C. Song, G. Psakis, J. Kopycki, C. Lang, J. Matysik, J. Hughes, J. Biol. Chem. 2014, 289, 2552-2562.
[21] H. Takala, A. Björling, O. Berntsson, H. Lehtivuori, S. Niebling, M. Hoernke, I. Kosheleva, R. Henning, A. Menzel, J. A. Ihalainen, S Westenhoff, Nature 2014, 509, 245-248.

[22] T. Mathes, J. Ravensbergen, M. Kloz, T. Gleichmann, K. D. Gallagher N. C. Woitowich, R. St. Peter, S. E. Kovaleva, E. A. Stojković, J. T. M. Kennis, J. Phys. Chem. Lett. 2015, 6, 239-243.

[23] A. T. Ulijasz, G. Cornilescu, C. C. Cornilescu, J. Zhang, M. Rivera, J. L. Markley, R. D. Vierstra, Nature 2010, 463, 250-254

[24] P. Altoè, T. Climent, G. C. De Fusco, M. Stenta, A. Bottoni, L. SerranoAndrés, M. Merchán, G. Orlandi, M. Garavelli, J. Phys. Chem. B 2009 113, 15067-15073.

[25] B. Durbeej, Phys. Chem. Chem. Phys. 2009, 11, 1354-1361.

[26] A. Strambi, B. Durbeej, Photochem. Photobiol. Sci. 2011, 10, 569-579.

[27] X. Zhuang, J. Wang, Z. Lan, J. Phys. Chem. B. 2013, 117, 1597615986.

[28] J. Dasgupta, R. R. Frontiera, K. C. Taylor, J. C. Lagarias, R. A. Mathies, Proc. Natl. Acad. Sci. U. S. A. 2009, 106, 1784-1789.

[29] O. Falklöf, B. Durbeej, J. Comput. Chem. 2013, 34, 1363-1374.

[30] M. A. Mroginski, S. Kaminski, D. von Stetten, S. Ringsdorf, W. Gärtner L.-O. Essen, P. Hildebrandt, J. Phys. Chem. B 2011, 115, 1220-1231.

[31] H. M. Senn, W. Thiel, Angew. Chem. Int. Ed. 2009, 48, 1198-1229.

[32] M. E. Casida, J. Mol. Struct. (THEOCHEM) 2009, 914, 3-18.

[33] W. D. Cornell, P. Cieplak, C. I. Bayly, I. R. Gould, K. M. Merz, Jr., D. M. Ferguson, D. C. Spellmeyer, T. Fox, J. W. Caldwell, P. A. Kollman, J. Am. Chem. Soc. 1995, 117, 5179-5197.

[34] J.-D. Chai, M. Head-Gordon, Phys. Chem. Chem. Phys. 2008, 10 6615-6620.

[35] T. Yanai, D. P. Tew, N. C. Handy, Chem. Phys. Lett. 2004, 393, 51-57.

[36] H. likura, T. Tsuneda, T. Yanai, K. Hirao, J. Chem. Phys. 2001, 115 , 3540-3544.

[37] D. Bakowies, W. Thiel, J. Phys. Chem. 1996, 100, 10580-10594.

[38] B. Durbeej, O. A. Borg, L. A. Eriksson, Chem. Phys. Lett. 2005, 416, 83-88.

[39] P. W. Kim, N. C. Rockwell, L. H. Freer, C.-W. Chang, S. S. Martin, J. C. Lagarias, D. S. Larsen, J. Phys. Chem. Lett. 2013, 4, 2605-2609.

[40] X. Xu, S. Gozem, M. Olivucci, D. G. Truhlar, J. Phys. Chem. Lett. 2013 4, 253-258.

[41] X. Yang, E. A. Stojković, W. B. Ozarowski, J. Kuk, E. Davydova, K Moffat, Structure 2015, 23, 1179-1189. 


\section{Entry for the Table of Contents}

\section{COMMUNICATION}

An outstanding question in current research on photoreceptor proteins is understanding why the primary photoisomerization event of phytochromes always occurs at the CD methine bridge of the linear tetrapyrrole chromophore, despite that other isomerization channels are available. Here, we demonstrate computationally that phytochromes effectively block the competing reaction channels by posing steric hindrance for their completion.

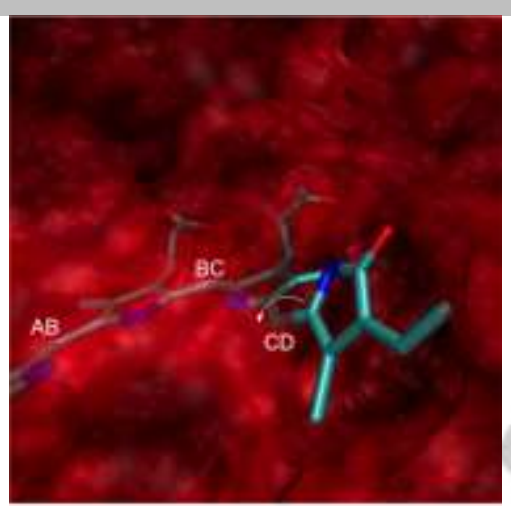

Olle Falklöf and Bo Durbeej*

Page No. - Page No.

Steric Effects Govern the Photoactivation of Phytochromes 